\title{
STUDIES ON BLOOD CHOLESTEROL IN SYPHILIS *
}

\author{
ALBERT R. MCFARLAND, M.D.
}

Assistant in Section on Dermatology and Syphilology and Fellow

in The Mayo Foundation

ROCHESTER, MINN.

This study was undertaken with the idea of determining, if possible, any relation between the blood cholesterol and the serologic and clinical manifestations of syphilitic infections.

Cholesterol, according to Hawk, ${ }^{1}$ is a monatomic alcohol containing at least one double bond and possessing the formula of $\mathrm{C}_{27} \mathrm{H}_{45} \mathrm{OH}$ or $\mathrm{C}_{27} \mathrm{H}_{43} \mathrm{OH}$. The exact formula is somewhat in dispute. It is soluble in ether, chloroform and benzene, and may be crystallized in thin transparent plates. Just as glycerol and the fatty acids unite to form glycerol fats, so cholesterol and fatty acids may form cholesterol fats. The cholesterol fats are more resistant to enzymic and bacterial action than the glycerol fats, and are thus found in the sebum of the skin and in the structural elements of red blood cells and tissue cells $\left(\right.$ Macleod $\left.^{2}\right)$.

SOURCE

Cholesterol probably has two origins, exogenous and endogenous; some writers maintain that it is entirely exogenous. It seems to be the consensus of opinion that most, but not all, of the body cholesterol is exogenous, as shown by Luden ${ }^{3}$ and others. Macleod has pointed out two factors that point toward its endogenous origin, namely, that the cholesterol in the feces of herbivorous animals is of the same variety as that in the feces of carnivorous animals and not phytosterol, which is present in plants, and that the universal presence of cholesterol in cells indicates that it must be manufactured there. Luden has studied extensively the effect of the ingestion of foods of high cholesterol value on the blood cholesterol. She has called attention to the fact that, since variations occur which depend on many factors, great care must

* From the Section on Dermatology and Syphilology, Mayo Clinic and The Mayo Foundation.

1. Hawk, P. B.: Practical Physiological Chemistry, Ed. 5, Philadelphia, P. Blakiston's Son \& Co., 1916, p. 638.

2. Macleod, J. J. R.: Physiology and Biochemistry in Modern Medicine, Ed. 3, St. Louis, C. V. Mosby Co., 1920, p. 99.

3. Luden, Georgine: Studies on Cholesterol. IV. Experiments Concerning the Relation of the Diet, the Blood Cholesterol, and the "Lymphoid Defense," J. Lab. \& Clin. Med. 3:141 (Dec.) 1917. 
be exercised in choosing foods of presumably high cholesterol content. For instance, milk from cows may be different at calving time from that at other periods. The cholesterol content of cheese may vary according to the time consumed in ripening, and so forth. Luden has, however, apparently demonstrated the fact that certain foods, such as eggs, cream and butter, are fairly high in cholesterol content. Through the ingestion of an exclusive meat diet, a definite rise in the blood cholesterol may be observed. On the other hand, by feeding foods poor in cholesterol, such as fruit, vegetables and milk, the blood cholesterol can be definitely lowered. Hawk is of the opinion that most of the cholesterol is exogenous, but that under special stress the body may be able to produce endogenous cholesterol.

\section{OCCLRRENCE}

Cholesterol is perhaps an almost universal constituent of the body tissues. It enters into the structural makeup of the cells. It is found most abundantly in the nervous tissue, liver and bile. It is found in the red blood cells, in the plasma and in the cerebrospinal fluid. It seems to be excreted largely in the bile, perhaps owing to the fact that the bile salts are a powerful solvent of cholesterol. It has been estimated that the bile itself contains about 1.6 parts of cholesterol for each 1,000 parts of fluid. This is probably an important factor in the production of gallstones.

\section{FUNCTION}

As has been stated, the cholesterol fats are resistant to the action of enzymes and bacteria. Hence, it is found in the sebum of the sebaceous glands and probably serves to protect the cutaneous glandular structures from the constant assault of these invaders. According to Macleod, it probably is an important element in the formation of the skeletal structure of blood and tissue cells. Kipp, ${ }^{4}$ Denis ${ }^{5}$ and others have shown that a definite relation exists between the blood cholesterol and the clinical course of such diseases as pneumonia and certain other acute infections. They have also called attention to the relationship of blood cholesterol to leukocytosis. The inference to be deducted is that the cholesterol plays an important part in the antitoxic and bactericidal powers of the body and probably is a factor in antibody formation. Luden, in a study of blood cholesterol in neoplasms, apparently has shown that there is a relation between the two. Whether cholesterol is utilized directly for the formation of cells or acts merely

4. Kipp, H. A.: Variation in the Cholesterol Content of the Serum in Pneumonia, J. Biol. Chem. 44:215 (Nov.) 1920.

5. Denis, W.: Cholesterol in Human Blood Under Pathological Conditions, J. Biol. Chem. 29:93 (Feb.) 1917. 
as a catalyst (as suggested by Robertson) is not definitely known, but in malignancy there is evidence of disturibances of cholesterol metabolism.

CHOLESTEROL VARIATIONS IN VARIOUS DISEASES

During the past few years, numerous cholesterol studies have been made in specific diseases and syndromes in an endeavor to throw additional light on the real nature and function of the blood cholesterol. It is only natural to surmise that a substance so widely distributed in the human economy may be intimately involved in the process of immunity and that quantitative estimations may prove to be of diagnostic if not of therapeutic importance in combating disease.

Wilensky and Rothschild ${ }^{6}$ have made a study of blood cholesterol in gallbladder diseases. As a result of a study of about seventy cases, they conclude: "It must be conceded that in any given case the diagnostic value of any determination of the cholesterol content of the blood is a variable and dubious one." They further conclude that when cholesteremia is found in a case of jaundice in which it is a question of whether the jaundice is obstructive or cirrhotic, the probabilities are that it is obstructive, provided no other conditions are present which may cause a cholesteremia, such as pregnancy, diabetes or nephritis.

Kipp, in 1920, in a rather extensive study of thirty-five cases of pneumonia and empyema, has found that during the period of invasion by organisms the blood cholesterol is low. As convalescence takes place, there is a gradual increase until cholesteremia obtains, the degree of which apparently depends on the virulence of the infection and the extent of pulmonary involvement. Several cases are cited in which the cholesterol values previous to death were very low. Henes, ${ }^{7}$ in a series of twelve cases of chronic nephritis, has obtained almost uniformly high blood cholesterol values, and expresses the opinion that a cholesteremia in such cases is of considerable diagnostic value. He has also found, in cases of typhoid fever, that during the acute stage the blood cholesterol is low; later it becomes normal, and during convalescence a cholesteremia results. This observation seems to agree with that of Kipp concerning his cases of pneumonia.

Schnabel ${ }^{8}$ studied a series of sixty cases of gastro-enterologic disorders. These included cases of pernicious anemia with gastric hyper-

6. Wilensky, A. O., and Rothschild, M. A.: Studies in Cholelithiasis. II. The Clinical Relationships of the Cholesteremia to the Pathologic Process, Am. J. M. Sc. 156:404 (Sept.) 1918.

7. Henes, E.: The Prognostic Value of Cholesteremia in Chronic Nephritis, Arch. Int. Med. $25: 411-419,1920$.

8. Schnabel, T. G. : Blood Cholesterol in Gastro-Enterologic Cases, Am. J. M. Sc. 160:42.3 (Sept.) 1920. 
acidity, catarrhal and obstructive jaundice, duodenal ulcer, intestinal stasis, chronic constipation, gastric neurosis, and chronic appendicitis. $\mathrm{He}$ concludes that when a number of cases are studied, the results are too variable to be of any practical value in gastro-enterologic diagnosis.

From these observations, it may be seen that in certain diseases there seems to be a definite relation between the blood cholesterol and the pathologic conditions, while in others no definite relation can be shown.

Certain phenomena concerning the pathology of syphilis suggested that blood cholesterol determinations might be of clinical significance. The Wassermann test, founded on the conception of the formation of specific antibodies, supported the assumption that Spirochaeta pallida, or more strictly speaking its chemical composition, produced a specific antibody, which in the presence of the complement, formed a stable combination; and thus the test was applied in the diagnosis of syphilis. It was not until it was demonstrated by Landsteiner, Levaditi, Noguchi and others, that substances beside spirochete-containing tissue could serve as an antigen in the complement-fixation test that the specificity of the Wassermann reaction came to be questioned. Among the various antigens which have been devised, one of the most delicate is the cholesterol treated alcoholic extract of heart tissue. This would seem to indicate that the Wassermann reaction of syphilis might possibly be due in part to the cholesterol content of the blood.

Going back a step, it may be noted that cholesterol is present to the greatest degree in nerve and liver tissue. These tissues are often attacked by Spirochaeta pallida, and hence we might believe that cholesterol would be liberated, resulting in cholesteremia, and therefore would have some bearing on the positive Wassermann reaction. On the other hand, I had an opportunity to verify the well-known relative insolubility of cholesterol while working on Wassermann technic in the clinical laboratories. The antigens were prepared by adding 0.04 per cent. of commercial cholesterol to an alcoholic extract of human, beef or guinea-pig heart. It was noted that, at room temperature, even this small amount formed a saturation so that free cholesterol settled to the bottom of the flask. Heating was necessary in order completely to dissolve it. Even with this apparently saturated solution, many specimens still were found to lack antigenic power and others proved to be only equal or slightly stronger than the ordinary acetone-insoluble alcoholic extract fraction which was used for routine work in the laboratory.

In spite of the fairly well standardized methods of determining blood cholesterol, writers have reported widely varied data. Widal, 
Weill and Laudat ${ }^{9}$ consider from 175 to $195 \mathrm{mg}$. for each 100 c.c. to be about normal. Luden places normal values at from 70 to $100 \mathrm{mg}$. for each 100 c.c., with the Bloor ${ }^{10}$ technic as used by her. Other investigators have reported data showing values from 50 to 450 in various conditions. The determinations in this series have all been made under the direction of Dr. Luden of the Mayo Clinic, with a uniformly standard technic, and by the Bloor I and Bloor II methods. While the data taken may not agree with those of other investigators, they should have a fair degree of uniformity for the cases studied.

\section{TECHNIC}

Two determinations were made on each specimen, one designated Bloor I and the other Bloor II. The chief difference between the two is that in the Bloor I method, sodium ethylate is added to the ether-alcohol extract in order to obtain the proper color for colorimetric determinations. In the Bloor II method, sodium ethylate is not used and the proper colorimetric qualities are maintained by the use of more care in heating the extract. The Bloor II values are as a rule higher than the Bloor I.

Five cubic centimeters of blood are withdrawn from the cubital vein by a large needle and syringe and immediately injected into 75 c.c. of an alcohol-ether mixture $(3: 1)$ which is then agitated for thorough mixing and heated just to boiling; the amount is then made up to 100 c.c. In the Bloor I method, 2 c.c. of sodium ethylate is added to each 10 c.c. of the extract. Evaporation to dryness is carried out on a water-bath and the residue is extracted three times with dry chloroform. To each 6 c.c. of the chloroform extract, 2 c.c. of acetic acid and 0.2 c.c. of concentrated sulphuric acid are added, in order to produce a green color. The test tubes are then exposed to the daylight for five minutes at a temperature of $20 \mathrm{C}$., in order to secure a color comparable to the standard. Calorimetric readings are made against a standard solution; each reading is checked repeatedly. A more detailed description of the technic may be obtained by referring to Luden's original article. By this method, it has been found that the normal Bloor I value is between 70 and $100 \mathrm{mg}$. for each 100 c.c.; values from 100 to 140 are increased; from 140 to 200 are high, and 200 or more are unusually high. The normal difference between the Bloor I and Bloor II values is from 17 to $34 \mathrm{mg}$. for each 100 c.c.

9. Widal, F.; Weill, A., and Laudat, M.: Comparative Study of the Contents of Free Cholesterol and Its Esters in Blood Serum, Compt. rend. Soc. de biol. 74:882, 1913.

10. Bloor, W. R.: Studies on Blood Fat. II. Fat Alssorption and the Blood Lipoids, J. Biol. Chem. 23:317, 1915; Determination of Cholesterol in Blood, J. Biol. Chem. 24:227 (March) 1916; Bloor, W. R., and Knudson, A.: Separate Determination of Cholesterol and Cholesterol Esters in Small Amounts of Blood, J. Biol. Chem. 27:107 (Oct.) 1916. 
The Bloor I value represents the unchanged cholesterol in the blood stream. The Bloor II value indicates the free cholesterol plus the combined cholesterol; the difference between the two represents roughly the rate at which cholesterol is being metabolized.

In this study, the cholesterol values are placed in inree groups. Anything above 140 is classified high, from 100 to 140 medium, and below 100 low. The difference between the Bloor I and Bloor II values has been divided into three groups; namely, values above 34 high, below 17 low, and the remainder normal.

WASSERMANN DETERMINATIONS

The Wassermann tests were made by Sanford. A few salient points in the technic may be mentioned. The raw serum is employed without heating. The antigen used is the acetone-insoluble alcoholic extract of beef heart. Mixed guinea-pig serum furnishes the complement. Human cells and antihuman dog amboceptor are used in the second incubation series.

\section{CONDITIONS OF THE EXPERIMENT}

The tests were made on syphilitic patients taken at random from the section on dermatology and syphilology. The original diagnosis of syphilis in each case had been determined by the Wassermann test, examination of the cerebrospinal fluid, dermatologic examination, and the general examination, including neurologic, cardiovascular, and roentgen-ray examinations if indicated. The tests were all made under conditions as nearly uniform as possible. Each patient had been at rest for from six to eight hours and had not had food for the same length of time. Most of the Wassermann tests were made on the same day as the cholesterol test. Some were made the week before or the week after, which for practical diagnostic purposes may be considered within reasonable limits; a few made several months before were discounted. Problems as follows were considered:

1. The relation, if any, between the Wassermann reaction and the blood cho'esterol.

2. The usual cholesterol value in cases of syphilis in general.

3. The relation between the cholesterol values and the progress of treatment; that is, the amount of arsphenamin already received.

4. The relation of the nearness of the last arsphenamin injection to the cholesterol values.

5. The constancy, if any, of difference between the Bloor I and Bloor II values in syphilitic patients.

6. The relation between the Wassermann reaction and the difference between Bloor I and Bloor II values. 
7. The relation between the clinical and serologic response of patients and the cholesterol findings.

8. The relation of the clinical type of the syphilitic infection to the cholesterol findings.

9. The constant change, if any, in cholesterol values of a patient during a course of treatment.

Among the high cholesterol values (above 140, Bloor I), there were two positive and nineteen negative Wassermann reactions (9.5 per cent. were positive). In the medium values (from 100 to 140 , Bloor I), there were twenty-two positive and forty negative reactions (25.4 per cent. were positive). In the low cholesterol values (below 100, Bloor I), there were five positive and fifteen negative Wassermann reactions ( 33.33 per cent. positive). In other words, of the twentynine patients with positive Wassermann reactions on the blood, 6.8 per cent. had high cholesterol values, 75.9 per cent. had medium cholesterol values, and 17.2 per cent. had low cholesterol values.

Thus it appears that the bulk of the positive Wassermann reactions are among the medium and low values, although in some cases high values occur. In general, it was found that of the 103 patients with syphilis, twenty-one (20.3 per cent.) had high cholesterol values, sixtytwo (60.3 per cent.) had medium values, and twenty (19.4 per cent.) had low values. Here again the bulk of the patients seem to fall into the low and medium groups, which might be expected in any chronic infective process. The recent work of Craig ${ }^{11}$ on injection of animals with cholesterol also corroborates these findings.

The patients with high cholesterol values had received an average of 8.9 injections of arsphenamin; those with medium values, 8.2 injections; and those with low values, 7.25 injections. The patients with high values had received from none to nineteen injections of arsphenamin; those with medium values, from none to twenty-eight injections; and those with low values, from one to seventeen injections. Examples of all stages of treatment were therefore included. There seems to be no relation between the amount of treatment and the cholesterol values.

A recent article by Strickler ${ }^{12}$ contained the observation that a large percentage of nonsyphilitic patients give positive Wassermann reactions after receiving arsphenamin. To explain this, it was suggested that perhaps the arsphenamin acted on the liver, increasing the formation of

11. Craig, C. F., and Williams, W. C.: Experimental Observations upon the Effect of Cholesteremia on the Results of the Wassermann Tests, Am. J. Syph. 5:392 (July 21) 1921 .

12. Strickler, A.; Munson, H. G., and Sidlick, D. M.: Positive Wassermann Test in Nonsyphilitic Patients After Intravenous Therapy. Preliminary Report of a Study of Its Significance. J. A. M. A. 75:1488 (Nov. 27) 1920. 
lipoid, and thereby produced a positive Wassermann reaction. According to our observations and those of Kilduffe, ${ }^{13}$ arsphenamin does not produce positive Wassermann reactions, and it seems evident that cholesterol is not one of the substances which are increased as the result of the administration of arsphenamin.

In considering the time relations between arsphenamin treatment and the cholesterol tests, it was found that the nearest previous injection varied from one week to ten months in the high values, from one week to eighteen months in the medium values, and from one week to four months in the low values. The proximity of the last arsphenamin injection, therefore, would seem to have no relation to the cholesterol values, although it must be stated that determinations were not made if the arsphenamin injection was nearer than one week to the cholesterol determination.

Fifty and seven-tenths per cent. of the entire series of patients had a high difference (above 34 ) between the Bloor I and Bloor II values; 34.6 per cent. had a normal difference (between 17 and 34 ), and 14.7 per cent. had a low difference (below 17). Twenty-seven and seventenths per cent. of the patients with high differences had positive Wassermann reactions; 53 per cent. with normal differences had positive Wassermann reactions, and 37.5 per cent. with low differences had positive Wassermann reactions. The bulk of the cases of syphilis, therefore, fall into the groups showing high and normal differences between Bloor I and Bloor II values. It is difficult to determine whether the positive Wassermann reactions tend to predominate in the groups with high or low differences, but certainly the majority seem to be within normal limits (17 to 34 ).

Cholesterol has been proposed as an element in the nonspecific mechanism of defense of the body. This mechanism is seldom recognized, but is a significant factor in the course of many chronic infections, and it seemed, therefore, that the possible influence of cholesterol as a defensive agent or index of nonspecific defense should be particularly conspicuous in a chronic infection such as syphilis. In recent studies by Tokuda, and by Bircher and myself, the globulin-albumin ratio, perhaps also to be included among the nonspecific defense indexes, was found to be considerably affected by syphilitic infection and by treatment. It was with the hope of detecting a similar rôle for cholesterol that the present study was begun.

Next, comparisons were made between the cholesterol values and the clinical and serologic responses of the patient. The cases were divided into three arbitrary groups. In Group 1, good response, were

13. Kilduffe, R. A.: Effect of Intravenous Administration of Arsphenamin, Neo-Arsphenamin and Mercury on the Wassermann Test in Normal Serums, J. A. M. A. 76:1489-1490 (May 28) 1921. 
placed patients who improved quite rapidly clinically and those whose blood easily became negative. In Group 2 were placed patients showing a fair response, who eventually obtained a fairly satisfactory result with regard both to their clinical symptoms and to their serologic findings, but who were rather resistant to treatment. Group 3 included the clinical "wrecks" and patients whose blood remained persistently positive in spite of apparently adequate treatment. The results of this classification can best be shown in tabulated form (Table 1).

Table 1.-Rfiation Between Clinical Response and Cholesterol Findings

\begin{tabular}{|c|c|c|c|c|c|}
\hline \multirow{3}{*}{$\begin{array}{c}\text { Clinical } \\
\text { and Serologic } \\
\text { Response }\end{array}$} & \multicolumn{5}{|c|}{ Percentage of Patients } \\
\hline & \multicolumn{3}{|c|}{$\begin{array}{l}\text { Cholesterol } \\
\text { Values }\end{array}$} & \multicolumn{2}{|c|}{$\begin{array}{l}\text { Difference Between } \\
\text { Bloor I and Bloor II Values }\end{array}$} \\
\hline & High* $^{*}$ & Mediumt & Low: & High & Low \\
\hline 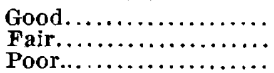 & $\begin{array}{l}15.9 \\
12.5 \\
25.0\end{array}$ & $\begin{array}{l}68.2 \\
55.0 \\
50.0\end{array}$ & $\begin{array}{l}15.9 \\
12.5 \\
25.0\end{array}$ & $\begin{array}{l}36.3 \\
41.6 \\
56.2\end{array}$ & $\begin{array}{l}63.7 \\
58.4 \\
43.8\end{array}$ \\
\hline
\end{tabular}

* High cholesterol, above 140 .

Medium cholesterol, 100 to 140

† Low cholesterol, below 100 .

While there is some variation in the percentages for the different groups, this is perhaps no more than might be expected from a percentage classification in a comparatively small series of cases. It might be concluded that a small difference between Bloor I and Bloor II values means a good prognosis, but this would need further confirmation before it could be accepted as a fact. As a whole, the cholesterol values do not furnish definite information regarding the probable prognosis in a given case. In Table 2 the basis for classifica-

TABle 2.-Relation Between Type of Syphilis and Cholesterol Findings

\begin{tabular}{|c|c|c|c|c|c|}
\hline \multirow[b]{3}{*}{ Type of Syphilis } & \multicolumn{5}{|c|}{ Percentage of Patients } \\
\hline & \multicolumn{3}{|c|}{$\begin{array}{c}\text { Cholesterol } \\
\text { Values }\end{array}$} & \multicolumn{2}{|c|}{$\begin{array}{c}\begin{array}{c}\text { Difference Between } \\
\text { Bloor I and Bloor II Values }\end{array} \\
\end{array}$} \\
\hline & High & Medium & Low & High & Normal and Low \\
\hline $\begin{array}{l}\text { Latent (positive Wassermann only) } \\
\text { Primary and secondary............ } \\
\text { Tertiary (osseous, visceral, cutane- }\end{array}$ & $\begin{array}{c}11.7 \\
0\end{array}$ & $\begin{array}{l}64.9 \\
77.7\end{array}$ & $\begin{array}{l}23.4 \\
22.3\end{array}$ & $\begin{array}{l}52.9 \\
55.5\end{array}$ & $\begin{array}{l}47.1 \\
44.5\end{array}$ \\
\hline 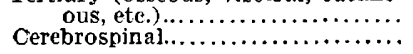 & $\begin{array}{c}0 \\
33.3\end{array}$ & $\begin{array}{l}80.3 \\
50.1\end{array}$ & $\begin{array}{l}17.7 \\
16.6\end{array}$ & $\begin{array}{l}47.0 \\
38.9\end{array}$ & $\begin{array}{l}53.0 \\
61.1\end{array}$ \\
\hline
\end{tabular}

tion is the type of syphilis instead of the clinical and serologic results. The noteworthy feature of this tabulation is the high percentage of syphilis of the central nervous system which is associated with high cholesterol values, namely, 33.3 per cent. as compared to 0.0 and 11.7 per cent. in the other types.

Most of the data obtained for this study are from single tests, taken on patients at random. However, in a series of twenty cases, deter- 
minations were made at weekly intervals during a course or part of a course of treatment, with the idea of detecting any possible constant variation from week to week (Table 3 ). This was suggested by the behavior of the globulin content of the serum in syphilitic patients under treatment, as observed by Bircher and myself. It may be seen that it is possible for a wide variation in the determinations to take place from week to week. This may be explained on the basis of some intercurrent infection of a nonspecific nature, although with few exceptions we were unable to locate such a factor. Certainly the variation cannot be explained on a basis of syphilis. This series of twenty cases included

TABle 3.-Chassification of Patients on Whom Determinations Were Made Weekly During a Course of Treatment

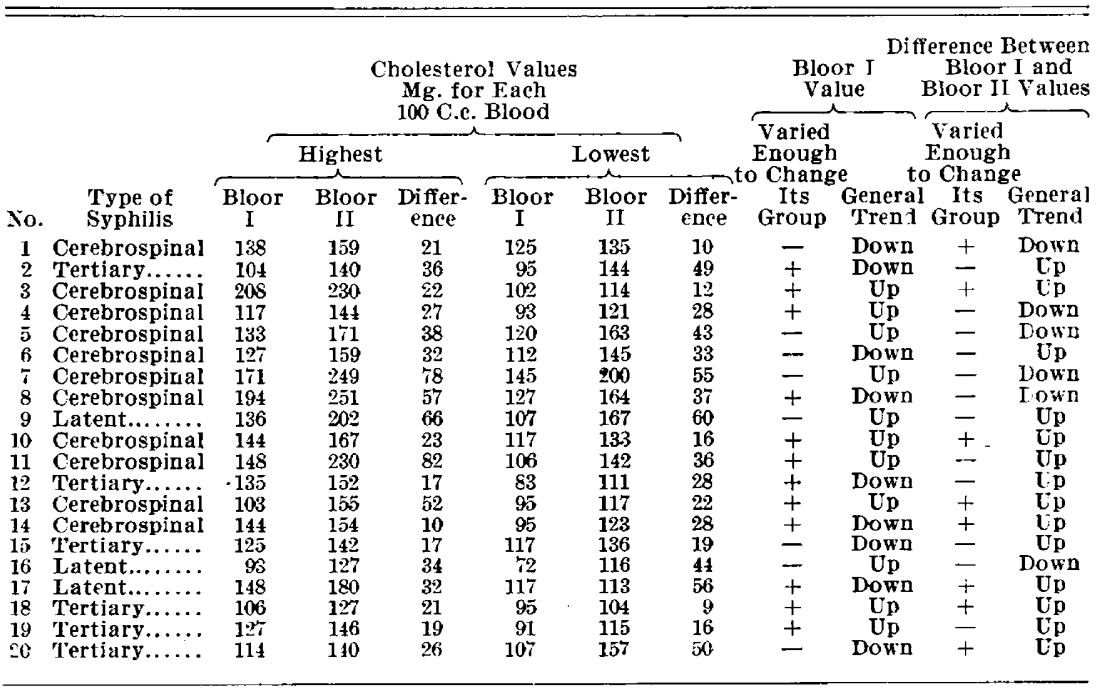

various types of syphilis. In twelve of the cases, the variation in Bloor I values was great enough from week to week to throw the result into a different group; that is, high, medium, or low. In eight of the twenty cases, the difference between the Bloor I and Bloor II values varied enough to change it from high to medium or to low. The general trend of the difference was up in fourteen instances and down in six instances. The general trend of the Bloor I values was down in nine instances and up in eleven. Treatment, therefore, in an individual case does not seem to result in any constant effect on the cholesterol values.

\section{CONCLUSIONS}

1. The positive Wassermann reaction on the blood apparently does not depend on high cholesterol value. While it is interesting to corrob- 
orate this fact, it is not surprising in view of the probable complexity of the Wassermann reaction.

2. Blood cholesterol values in syphilitic patients, in general, tend to be medium and low rather than high. The occasional high values might possibly be explained on some other basis, but no attempt was made to determine this, since the study was confined to syphilis. This may mean that cholesterol as an agent of defense is low in a chronic disease like syphilis, just as leukocytosis is low in chronic infections; but from a practical standpoint it cannot be said that the cho'esterol determinations, with the possible exception of findings in the cases of involvement of the central nervous system, have afforded results much different from those to be expected from a similar series of nonsyphilitic patients.

3. The amount of arsphenamin given and the time between injections do not obviously affect the blood cholesterol values. If cholesterol is an agent of defense in syphilis, it has not been detected in most cases in greater amount than might be expected in a series of average nonsyphilitic persons, and hence it is not affected materially by treatment for syphilis.

4. The majority of syphilitic patients have high or normal differences between the Bloor I and Bloor II values, while only a few have low differences; the significance of this has not been determined.

5. There is, apparently, no relation between the cholesterol values and the clinical and serologic response of the patient.

6. The only recognizable relation between the clinical type of syphilis and blood cholesterol values is the large proportion of high cholesterol estimations in syphilis of the central nervous system. 